\title{
Experimental and numerical analysis of clamped joints in front motorbike suspensions
}

\author{
D. Croccolo ${ }^{1, a}$, M. De Agostinis ${ }^{1}$, N. Vincenzi ${ }^{1}$ \\ ${ }^{1}$ DIEM, Faculty of Engineering, University of Bologna, V.le Risorgimento 2, 40136 Bologna, Italy
}

\begin{abstract}
Clamped joints are shaft-hub connections used, as an instance, in front motorbike suspensions to lock the steering plates with the legs and the legs with the wheel pin, by means of one or two bolts. The preloading force, produced during the tightening process, should be evaluated accurately, since it must lock safely the shaft, without overcoming the yielding point of the hub. Firstly, friction coefficients have been evaluated on "ad-hoc designed" specimens, by applying the Design of Experiment approach: the applied tightening torque has been precisely related to the imposed preloading force. Then, the tensile state of clamps have been evaluated both via FEM and by leveraging some design formulae proposed by the Authors as function of the preloading force and of the clamp geometry. Finally, the results have been compared to those given by some strain gauges applied on the tested clamps: the discrepancies between numerical analyses, the design formulae and the experimental results remains under a threshold of $10 \%$.
\end{abstract}

\section{Introduction}

Clamped joints are shaft-hub connections used, as an instance, in front motorbike suspensions to lock the steering plates with the legs (fork clamp) and the legs with the wheel pin (wheel clamp) by means of one or two bolts. The fundamental design parameter for this type of couplings is the preloading force, produced during the tightening process: it should be accurately evaluated since it must lock safely the shaft (legs or wheel pin) without overcoming the yielding point of the hub (fork or wheel clamp). Bolts are normally tightened by means of a calibrated torque wrench so that the preloading force results as a function of the total applied torque, of the screw geometry and of the friction coefficients (underhead friction and thread friction). Friction coefficients, which can typically assume values included in the range $0.05-0.5$, are known to be strongly influenced by several parameters, such as type of contact, surface finishing, lubrication, wear and spoiling. As a matter of fact the same tightening torque produces a high locking force if friction coefficients are low; conversely, high friction coefficients could provide an insufficient locking force. Therefore an incorrect evaluation of friction parameters may lead to dangerous failures [1], especially in applications where human safety is involved, such as front motorbike suspensions.

In previous works [2, 3], Croccolo et al. investigated the tensile state of clamped joints in front motorbike suspensions, via FEM: they developed and proposed some engineering design formulae, in order to estimate the maximum stress on the clamp and the mean coupling pressure as functions of the bolt preloading force and of the clamp geometry. The present work deals with the extension of

a e-mail : dario.croccolo@unibo.it

This is an Open Access article distributed under the terms of the Creative Commons Attribution-Noncommercial License 3.0, which permits unrestricted use, distribution, and reproduction in any noncommercial medium, provided the original work is properly cited. 
the results presented in Ref.[2, 3] using, as loading input parameter for the calculation, the tightening torque (in the place of the preloading force), which is actually controlled during the manufacturing process.

\section{Methodology}

Firstly the well known relationships (Eq.(1a,b) [4]) between the applied tightening torque $T$ [Nmm] and the imposed preloading force $F_{V}[\mathrm{~N}]$ have been studied, by applying the Design of Experiment (DOE) approach $[5,6]$ in order to evaluate the friction parameters accurately. A full factorial plane, characterized by 4 variables with 2 levels each, has been designed. Three replicas have been carried out, in order to reduce the influence of noise (experimental error) and of any non-investigated factors: a total of $3 \cdot 2^{4}=48$ experimental tests have been run. The levels include cast versus forged aluminium alloy, anodized versus spray-painted surfaces, lubricated versus dry screws and first tightening (fresh unspoiled surfaces) versus sixth tightening (spoiled surfaces) [5]. In Tab.1 the DOE parameters are summarized.

$$
\begin{aligned}
& \text { (a) } T=F_{V} \cdot\left[0.16 \cdot p+0.58 \cdot \mu_{m} \cdot d_{2}+0.5 \cdot \mu_{m} \cdot d_{u}\right] \\
& \text { (b) } T=K \cdot F_{V} \cdot d
\end{aligned}
$$

$p[\mathrm{~mm}]$ is the thread pitch, $d[\mathrm{~mm}]$ is the nominal thread diameter, $\mu_{m}$ is the overall friction coefficient according to $[4,7], d_{2}[\mathrm{~mm}]$ is the mean thread diameter $\left(d_{2}=d-0,6495 \cdot p\right), d_{u}[\mathrm{~mm}]$ is the underhead mean diameter and $K$ is the nut factor $[8,9]$.

Table 1. The Design of Experiment (DOE) parameters: variables and levels.

\begin{tabular}{|c|c|c|}
\hline Variable & Low Level (0) & High level (1) \\
\hline A. Lubrication & Dry & Lubricated \\
\hline B. Process & Cast & Forged \\
\hline C. Surface finishing & Spray-painted & Anodized \\
\hline D. Tightening & First tightening (unspoiled surfaces) & Sixth tightening (spoiled surfaces) \\
\hline
\end{tabular}

Some "ad hoc designed" specimens reported in Fig.1 [5] have been used, according to Eq. (2a,b), to calculate 48 different values of the overall friction coefficient $\mu_{m}$ and of the nut factor $K: T$ is given by a torque wrench, whereas $F_{V}$ has been evaluated by means of a strain gauge, located on the external surface of the specimen, which is able to provide the axial compression strain $\varepsilon_{C}$. The compression force acting on the specimen is equal, in magnitude, to the preloading force acting on the screw, since the system works like series of mechanical stiffness during the tightening phase. The whole cross section (tubular) in the central part of the specimen ("calibrated length" in Fig.1), has the same strain and, therefore, the same stress. Thus, it is possible to calculate the compression force acting on the specimen, which is the same tensile force (preloading) acting on the screw. The study considers M8x1.25, SAE Standard 8.8 galvanized screws.

$$
\begin{aligned}
& \text { (a) } \mu_{m}=\frac{T / F_{V}-0.16 \cdot p}{0.58 \cdot d_{2}+0.5 \cdot d_{u}} \\
& \text { (b) } K=\frac{T}{F_{V} \cdot d}
\end{aligned}
$$


The Analysis of Variance (ANOVA) statistical approach has been then applied to the results in order to obtain the mathematical equations of friction coefficient $\mu_{m}$ (Eq.3) and nut factor $K$ (Eq.4) as functions of the investigated variables (or their interactions) which are actually significant in changing the values [5].
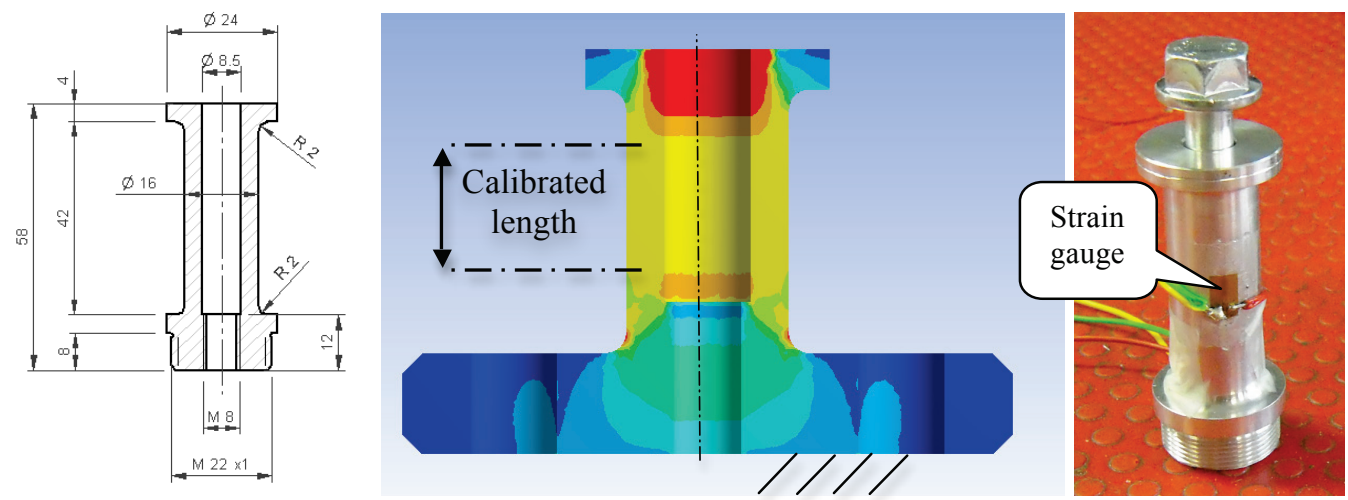

Fig.1 The specimen useful for relating the tightening torque $T$ to the preloading force $F_{V}$

$$
\begin{aligned}
& \mu_{\mathrm{m}}=0.108-0.023 \cdot A+0.103 \cdot C+0.010 \cdot D-0.007 \cdot A \cdot B-0.063 \cdot A \cdot C+ \\
& +0.017 \cdot A \cdot D+0.053 \cdot B \cdot C+0.117 \cdot C \cdot D-0.153 \cdot A \cdot C \cdot D \\
& K=0.157-0.028 \cdot A+0.123 \cdot C+0.013 \cdot D-0.008 \cdot A \cdot B-0.075 \cdot A \cdot C+ \\
& +0.015 \cdot A \cdot D+0.065 \cdot B \cdot C+0.138 \cdot C \cdot D-0.180 \cdot A \cdot C \cdot D
\end{aligned}
$$

As highlighted by equations (3) and (4), friction conditions are strongly affected by surface finishing ( $C$ variable), lubrication ( $A$ variable) and number of tightening and loosening ( $D$ variable); conversely, the forming process (cast or forged aluminium alloy) seems to have no significant influence on friction conditions [5]. Spray-painted specimens $(C=0)$ present the lower values of $\mu_{m}$ and $K$ (the higher preloading forces $F_{V}$ ). Lubrication $(A=1)$ always increases the preloading forces, while the tightening replicas $(D=1)$ progressively decrease the preloading forces, mainly in case of dry surfaces, as demonstrated also in Ref.[10].

Secondly the outcomes of the present analysis have been applied to an unlubricated, spray-painted, cast aluminium wheel clamp, realized by the Paioli Meccanica S.p.A. of Bologna (IT), which produces front motorbike suspensions. Two M6x1, SAE Standard 8.8 galvanized screws $(d=6 \mathrm{~mm}$, $p=1 \mathrm{~mm}, d_{2}=5.35 \mathrm{~mm}$ and $d_{u}=8 \mathrm{~mm}$ ), have been tightened up to six times each. Strain gauges have been placed on the critical section of the clamp (loaded by a bending stress, as deeply demonstrated in [2]), where a stress concentration factor $K_{t}$ occurs, mainly due to the presence of spot-facings and holes, as reported in Fig.2.
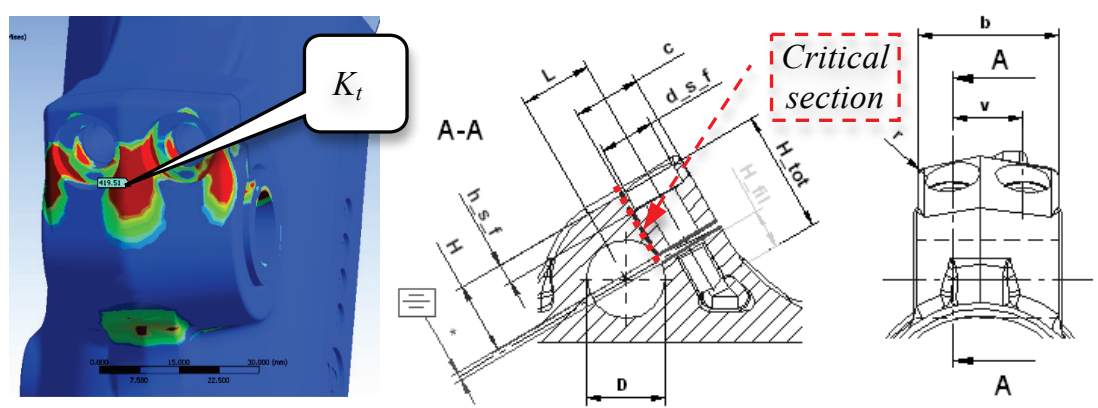

Fig.2 Example of wheel clamp: stress concentration factor and geometrical dimensions 
Referring to Fig.2, the theoretical bending stress in the critical section can be evaluated by applying Eq.(5) [2], in which $F_{V}$ is the preloading force, $a[\mathrm{~mm}]$ the lever arm of $F_{V}$ with respect to the critical section, $n$ the number of bolts, $b[\mathrm{~mm}]$ and $h[\mathrm{~mm}]$ are the width and the height of the rectangular cross section, respectively.

$$
\begin{gathered}
\sigma_{b_{-} t h}=\frac{M_{b}}{W_{b}}=\frac{n \cdot F_{V} \cdot a}{b \cdot h^{2} / 6} \\
\left\{\begin{array}{l}
\sigma_{b_{-} \max }=\sigma_{b_{-} t h} \cdot K_{t}=\frac{n \cdot F_{V} \cdot a}{b \cdot h^{2} / 6} \cdot K_{t} \\
K_{t \_M 6}=2.438+0.548 \cdot \frac{h_{s-f}}{h}-1.131 \cdot \frac{a}{d_{s-f}}-0.393 \cdot \frac{v}{d_{s-f}}
\end{array}\right.
\end{gathered}
$$

\section{Results}

The maximum bending stress on the clamp can be calculated by applying Eq.(6) in which the stress concentration factor $K_{t}$ takes into account the perturbation produced by the spot-facings located close to the coupling zone. The geometrical dimensions of clamps under investigation are reported in Tab.2: the actual $K_{t}$ value calculated applying Eq.(6) is equal to $1.37[2,3]$.

Table 2. Wheel clamp geometrical dimensions useful for the calculation according to Fig.2

\begin{tabular}{|c|c|c|}
\hline Description & Symbol & Value \\
\hline Wheel pivot diameter & $D[\mathrm{~mm}]$ & 20 \\
\hline Distance between the wheel pivot centre and the bolt axis & $L[\mathrm{~mm}]$ & 15.5 \\
\hline Lever arm of the preloading force $(L-D / 2)$ & $a[\mathrm{~mm}]$ & 5.5 \\
\hline Distance between the wheel pivot and the clamp side & $c[\mathrm{~mm}]$ & 16 \\
\hline Spot-facing diameter & $d_{s-f}[\mathrm{~mm}]$ & 11 \\
\hline Clamp total height & $h_{\text {tot }}[\mathrm{mm}]$ & 45 \\
\hline Clamp height & $h[\mathrm{~mm}]$ & 18.5 \\
\hline Thread height & $h_{\text {th }}[\mathrm{mm}]$ & 24.5 \\
\hline Spot-facing height & $h_{s-f}[\mathrm{~mm}]$ & 4.3 \\
\hline Clamp width & $b[\mathrm{~mm}]$ & 36 \\
\hline Distance between bolt axes & $v[\mathrm{~mm}]$ & 17.5 \\
\hline Mill radius & $r[\mathrm{~mm}]$ & 2 \\
\hline
\end{tabular}

A tightening torque $T=9.5 \mathrm{Nm}$ has been applied to the wheel clamp: the preloading forces produced by the tightening have been evaluated by means of Eq.(7), in which the overall friction coefficient $\mu_{m}$ is equal to 0.108 during the first tightening $(A=B=C=D=0$, according to Tab.1 and Eq.3) and equal to 0.118 during the sixth tightening $(A=B=C=0, D=1$, according to Tab.1 and Eq.3).

$$
F_{V}=\frac{T}{\left[0.16 \cdot p+0.58 \cdot \mu_{m} \cdot d_{2}+0.5 \cdot \mu_{m} \cdot d_{u}\right]}
$$

By applying the Von Mises equivalent stress criterion, the maximum preloading forces, with respect to the bolt yielding or failure, can be evaluated by means of Eq.(8) (see also the sketch reported in Fig.3), in which $S_{Y}[\mathrm{MPa}]$ is the yielding point and $S_{U}[\mathrm{MPa}]$ is the ultimate point of the screw (SAE Standard 8.8: $S_{Y}=640 \mathrm{MPa}, S_{U}=800 \mathrm{MPa}$ ), while $A_{t}\left[\mathrm{~mm}^{2}\right]$ is the screw tensile stress area and $d_{t}$ its diameter $\left(A_{t}=20.1 \mathrm{~mm}^{2}, d_{t}=5.06 \mathrm{~mm}\right.$, in case of bolt M6x1) [4]. 


$$
\begin{aligned}
& F_{V_{-} \text {yielding }}=\frac{S_{Y}}{\sqrt{\left(\frac{1}{A_{t}}\right)^{2}+3 \cdot\left(\frac{0.16 \cdot p+0.58 \cdot \mu_{m} \cdot d_{2}}{\frac{\pi \cdot\left(d_{t}\right)^{3}}{16}}\right)^{2}}} \\
& F_{V_{-} \text {failure }}=\frac{S_{U}}{\sqrt{\left(\frac{1}{A_{t}}\right)^{2}+3 \cdot\left(\frac{0.16 \cdot p+0.58 \cdot \mu_{m} \cdot d_{2}}{\frac{\pi \cdot\left(d_{t}\right)^{3}}{16}}\right)^{2}}}
\end{aligned}
$$

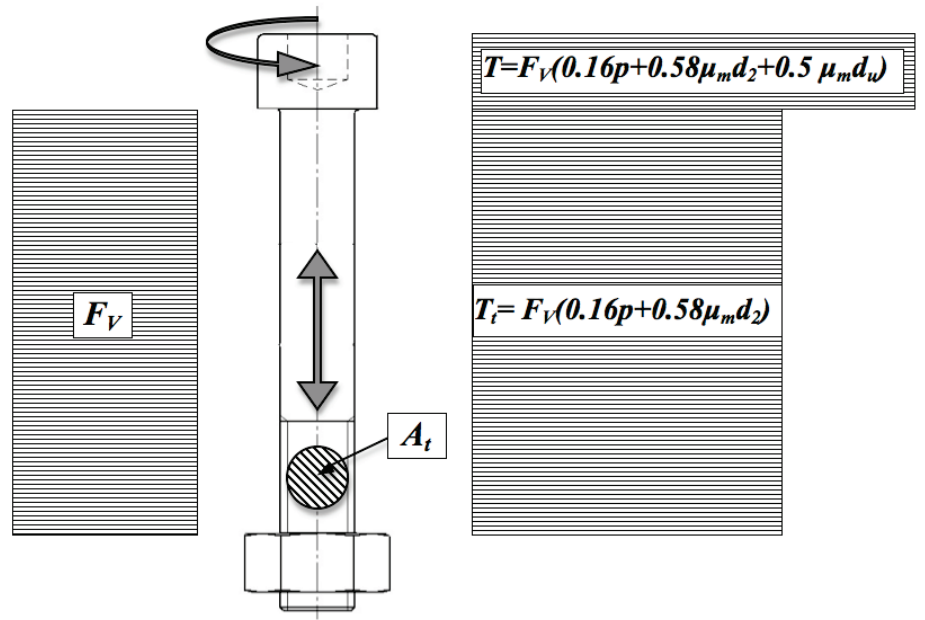

Fig.3 Sketch of the load diagrams (axial and torsional) acting on the bolt during the tightening

The numerical values of the preloading forces are reported in Tab.3, as a function of the overall friction coefficient $\mu_{m}$ (Eq.3).

Table 3. Preloading force values as a function of the overall friction coefficient (bolt: M6x1, 8.8)

\begin{tabular}{|c|c|c|c|c|c|}
\hline Clamp parameters $(\mathrm{Tab} .1)$ & $\boldsymbol{T} \mathbf{~ [ N m ]}$ & $\mathbf{a}_{m}$ & $\boldsymbol{F}_{\boldsymbol{V}}[\mathbf{k N}]$ (Eq.7) & $\boldsymbol{F}_{V_{-} \text {vielding }}[\mathbf{k N}](\mathrm{Eq} .8)$ & $\boldsymbol{F}_{\boldsymbol{V} \text { failure }}[\mathbf{k N}](\mathrm{Eq} .8)$ \\
\hline$A=B=C=D=0$ & 9.5 & 0.108 & 10.223 & 10.641 & 13.301 \\
\hline$A=B=C=0, D=1$ & 9.5 & 0.118 & 9.497 & 10.430 & 13.038 \\
\hline
\end{tabular}

During the first tightening (fresh and unspoiled surfaces) the strain gauge applied on the clamp (Fig.4), gives a strain value that is equivalent to a stress of 79MPa. A numerical (FEM) nonlinear analysis, with contact elements between the shaft and the hub, has been performed on the same joint by imposing the preloading force accurately calculated by the theoretical formulae $\left(F_{V}=10.223 \mathrm{kN}\right.$ of Tab.3). The stress evaluated via FEM is equal to $82 \mathrm{MPa}$, as shown in Fig.4, so that the difference between the experimental test and the FEM values is lower than $4 \%$. During the sixth tightening (spoiled surfaces) the test provides a strain value equivalent to a stress of $73 \mathrm{MPa}$ : the ratio between the sixth and the first tightening bending stresses $(73 / 79=0.924)$ is very close to the ratio between the first and the sixth preloading force calculated by the theoretical formulae $(9.497 / 10.223=0.929)$. 

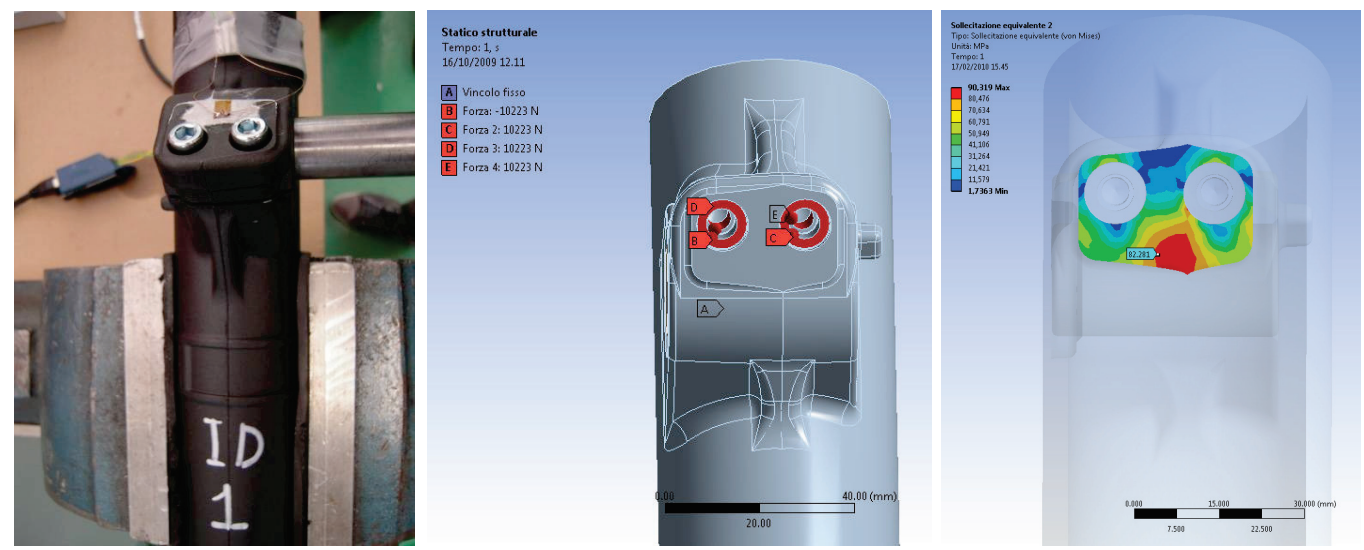

Fig.4 Experimental tightening test $(T=9.5 \mathrm{Nm})$ vs FEM analysis $\left(F_{V}=10.223 \mathrm{kN}\right)$

The engineering design formulae of Ref.[2], reported in Eq.(5) and (6), are able to provide, during the first tightening, a maximum bending stress $\sigma_{b \_ \text {max }}$ equal to $75 \mathrm{MPa}$ (theoretical bending stress $\sigma_{b \_t h}$ equal to $55 \mathrm{MPa}$ and stress concentration $K_{t}$ equal to 1.37 ): the discrepancy with respect to the experimental test value is equal to $5 \%$. The aforementioned findings are summarized in Tab.4.

Another clamp with the same geometry has been studied during the tightening phase: the bolt has been tightened until its failure, in correspondence of the sixth tightening. Since the yielding of the screw has been overcome, the torque-preloading relationship (Eq.7) is no more effective. However, according to Eq.(8), it is possible to calculate the preloading force in correspondence of the bolt failure, which is equal to $13.038 \mathrm{kN}$ (Tab.3). When the bolt failure occurs (Fig.5) the strain gauge applied on the clamp gives a strain value equivalent to a stress of $107 \mathrm{MPa}$.

Table 4. Comparison between the values provided by the experimental (strain gauge), the numerical (FEM) and the design formulae

\begin{tabular}{|c|c|c|c|c|c|}
\hline $\begin{array}{c}\text { Clamp parameters } \\
\text { (Tab.1) }\end{array}$ & $\begin{array}{c}\boldsymbol{T} \\
{[\mathrm{Nm}]}\end{array}$ & $\begin{array}{c}\boldsymbol{F}_{V} \\
{[\mathrm{kN}]}\end{array}$ & $\begin{array}{c}\boldsymbol{\sigma}_{\text {strain_gauge }} \\
{[\mathrm{MPa}]}\end{array}$ & $\begin{array}{c}\boldsymbol{\sigma}_{F E M}[\mathrm{MPa}] \\
(\mathrm{e} \%)\end{array}$ & $\begin{array}{c}\boldsymbol{\sigma}_{\text {design_formulae }}[\mathrm{MPa}] \\
(\mathrm{e} \%)\end{array}$ \\
\hline$A=B=C=D=0$ & 9.5 & 10.223 & 79 & $82(+4 \%)$ & $75(-5 \%)$ \\
\hline$A=B=C=0, D=1$ & 9.5 & 9.497 & 73 & $76(+4 \%)$ & $70(-4 \%)$ \\
\hline
\end{tabular}
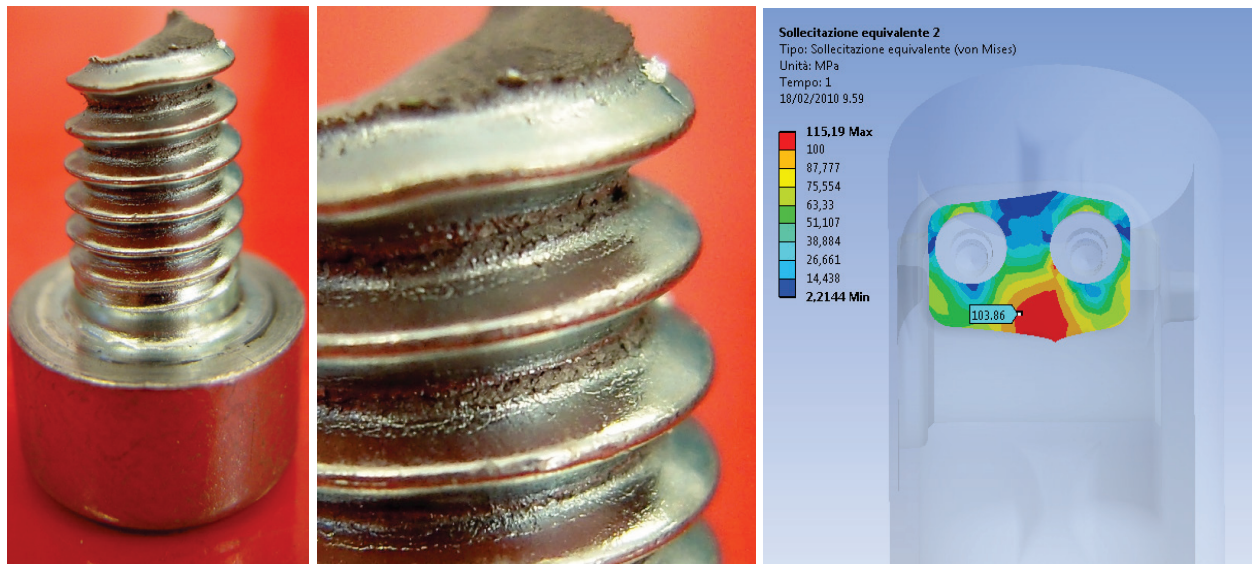

Fig.5 Experimental failure of a bolt, during the tightening phase 
By performing again the numerical nonlinear analysis on the joint and imposing the failure preloading force $F_{V_{\text {failure }}}=13.038 \mathrm{kN}$ a bending stress equal to $104 \mathrm{MPa}$ is reached, as shown in Fig.4: the discrepancy with respect to the experimental result is equal to $3 \%$. The engineering design formulae (Eq.6) provide a maximum bending stress $\sigma_{b \_ \text {max }}$ equal to $96 \mathrm{MPa}$ : the discrepancy with respect to the experimental result is equal to $10 \%$.

Finally, an original Software, Front Suspension Design $\left(\mathcal{C}\right.$, realised by the authors in Visual Basic ${ }^{\circledR}$ programming language [3], has been updated with the presented findings. The Software is a useful tool for the designing and the validation phases of any type of clamps in front motorbike suspensions. It is possible to calculate quickly the maximum bending stress on the clamp and the mean coupling pressure (output window in Fig.7) by simply inserting the total applied torque $T$, the clamp geometry and the production parameters of the clamp (input window in Fig.6) that are useful to evaluate the actual friction coefficient and, therefore, the actual preloading force.

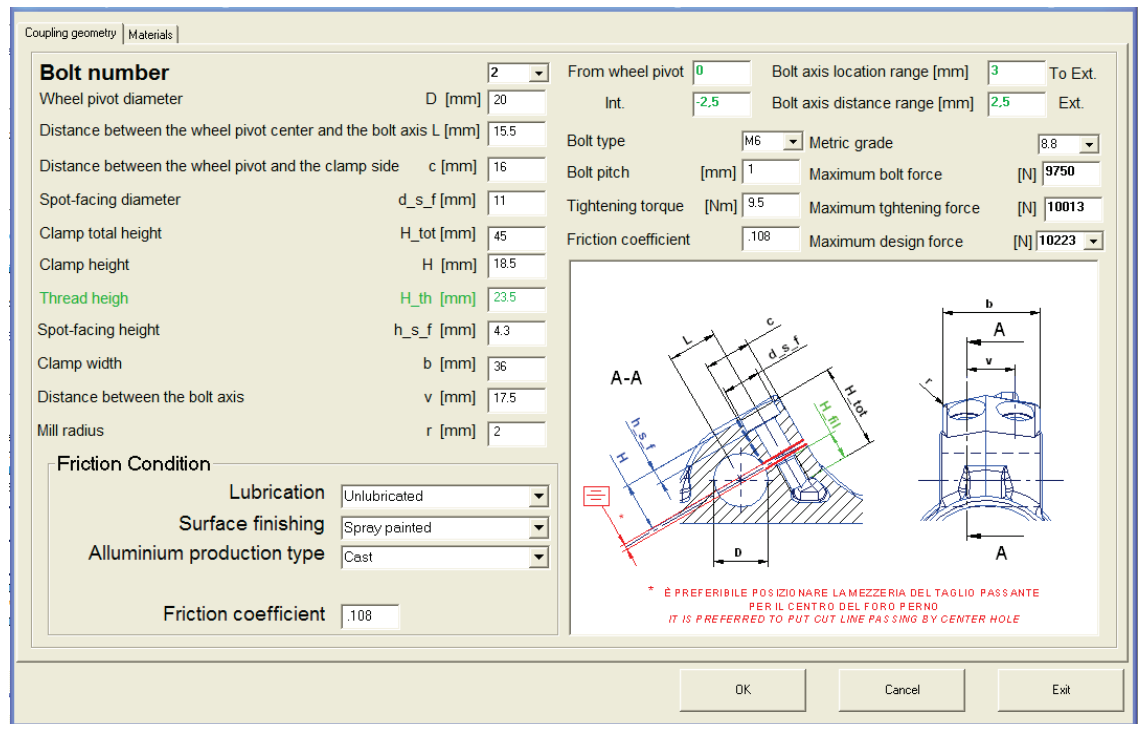

Fig.6 Input windows of Front Suspension Design $(\mathcal{C}$

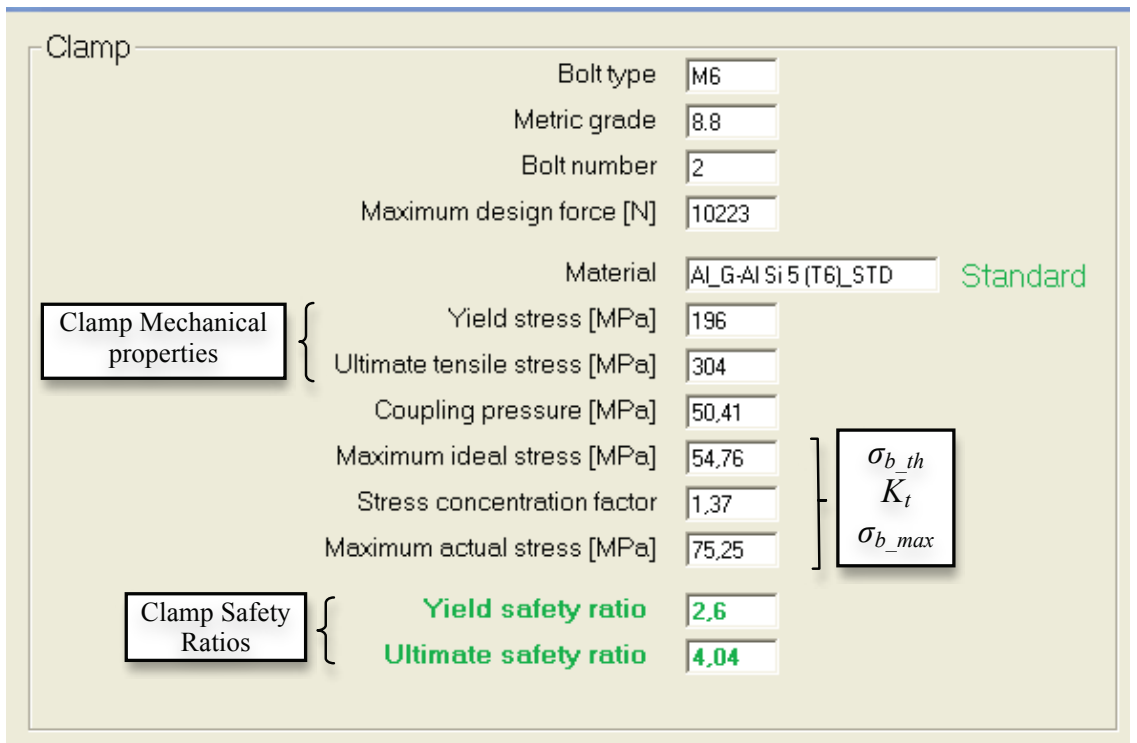

Fig.7 Output windows of Front Suspension Design $\odot$ 


\section{Conclusions}

Experimental tightening tests on clamped joints used in front motorbike suspensions have been studied in the present work. The maximum bending stress on the clamp, generated during the tightening, has been evaluated by applying some strain gauges on the actual component. The tightening has been performed by means of a calibrated torque wrench. In order to calculate the corresponding preloading force the overall friction coefficient of the joint has been calculated on some specific specimens, designed and realised with the same process and surface finishing of the actual components.

The experimental bending stresses obtained by the strain gauges have been compared both with those provided by some numerical nonlinear analyses and by some design formulae proposed and developed by the Authors. The input parameter for the experimental tightening is the tightening torque applied to the screw by a torque wrench, whereas the input parameter for the numerical simulation and for the design formulae application is the preloading force. By leveraging the accurate definition of the tightening torque-preloading force relationship the discrepancies in evaluating the maximum bending stress between experimental tests, numerical analyses and design formulae remains under a threshold of $10 \%$, both in the elastic and in the elastic-plastic field for the bolt.

In light of the proposed results, it is possible to estimate precisely the preloading force starting from the tightening torque and without performing any experimental tests. Furthermore it is possible to evaluate accurately the maximum stress generated on the clamp by applying the proposed design formulae: it is, therefore, easy to relate the applied tightening torque directly to maximum clamp stress.

An original software, Front Suspension Design $\mathbb{C}$, realised by the authors in Visual Basic ${ }^{\circledR}$ programming language, has been finally updated with the presented results. The Software is a useful tool for the designing and the validation phases of any clamps in front motorbike suspensions.

\section{References}

1. N. Eliaz, G. Gheorghiu, H. Sheinkopf, O. Levi, G. Shemesh, A. Ben-Mordechai, H. Artzi. Engineering Failure Analysis 10, 443-451(2003).

2. D. Croccolo, R. Cuppini, N. Vincenzi, Finite Elements in Analysis and Design 45, 406-414 (2009).

3. D. Croccolo, M. De Agostinis, N. Vincenzi, Engineering Failure Analysis DOI: 10.1016/j.engfailanal.2010.02.002, in press (2010).

4. VDI Handbuch Konstruktion 2230, Systematic calculation of high duty bolted joints - Joints with one cylindrical bolt (2001).

5. D. Croccolo, M. De Agostinis, N. Vincenzi, Experimental study of friction in aluminium bolted joints, $14^{\text {th }}$ International Conference on Experimental Mechanics, paper n.262, Poitiers, France (2010).

6. D. Croccolo, R. Cuppini, N. Vincenzi, Strain 44, 170-179 (2008)

7. DIN946, Determination of coefficient of friction of bolt/nut assemblies under specified conditions (1991).

8. S.A. Nassar, P.H. Matin, Journal of Pressure Vessel Technology 127, 387-393 (2005).

9. J.H. Bickford, An Introduction to the Design and Analysis of Bolted Joints, 3rd ed., Marcel Dekker, New York (1997).

10. W. Eccles, I.Sherrington, R.D.Arnell, Tribology International 43, 700-707 (2010). 